\title{
Photonic Crystal Cavities and Waveguides
}

\author{
A. Scherer, O. Painter, J. Vuckovic, M. Loncar, T. Yoshie \\ Professor of Electrical Engineering and Applied Physics, Caltech \\ MC 200-36, Pasadena CA 91125 \\ D. Dapkus, I. Kim \\ University of Southern California \\ T. Pearsall \\ CERF Corning, Avon France
}

Recently, it has also become possible to microfabricate high reflectivity mirrors by creating two- and three-dimensional periodic structures. These periodic "photonic crystals" can be designed to open up frequency bands within which the propagation of electromagnetic waves is forbidden irrespective of the propagation direction in space and define photonic bandgaps $[2,3]$. When combined with high index contrast slabs in which light can be efficiently guided, microfabricated two-dimensional photonic bandgap mirrors provide us with the geometries needed to confine and concentrate light into extremely small volumes and to obtain very high field intensities $[4,5]$. Here we show the use of these "artificially" microfabricated crystals in functional nonlinear optical devices, such as lasers, modulators, and waveguides.
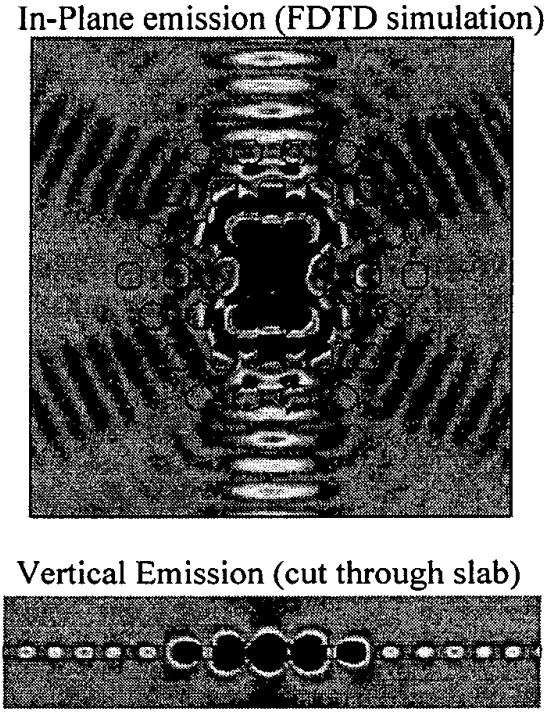

Figure 1. Field Intensity of light leakage from a single defect surrounded by 3 periods of photonic crystal and slightly enlarged holes in the horizontal direction. Light preferentially emits in the $y$ dipole.

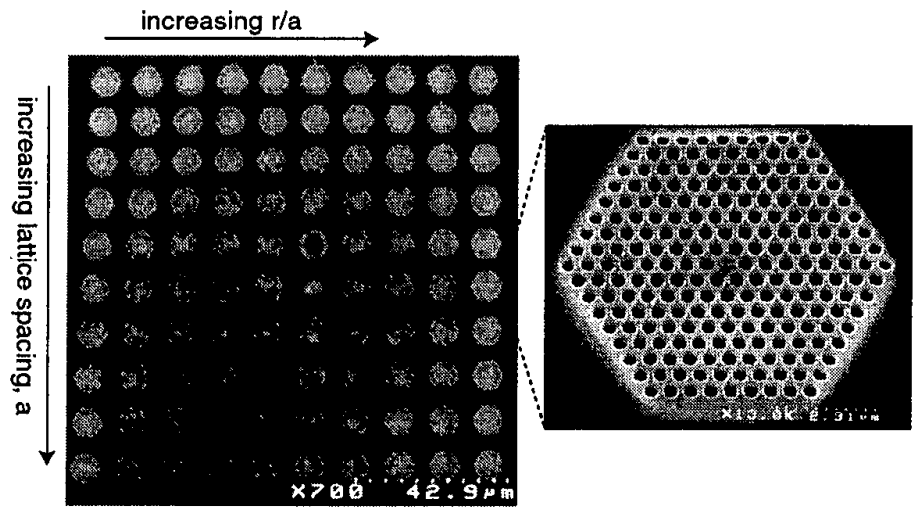

Figure 2. 10x10 multi-wavelength laser array within a 100x100 micron area. Each laser emits at a lithographically controlled
Sub-wavelength nano-optic cavities can be used for efficient and flexible control over both emission wavelength and frequency. Similarly, nanofabricated optical waveguides can be used for efficient coupling of light between devices. This new capability allows the reduction of the size of optical components and leads to their integration in large numbers, much in the same way 


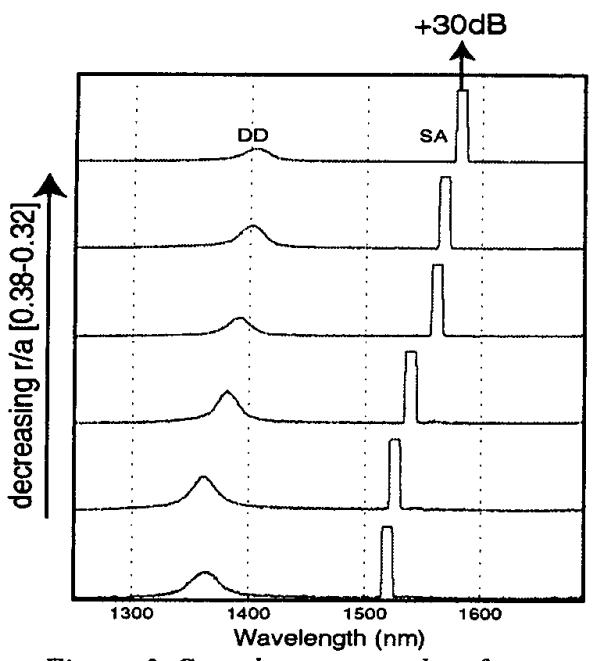

Figure 3. Sample spectra taken from lasers defined within the $10 \times 10$ array

as electronic components have been integrated for improved functionality to form microchips. As high-Q optical and electronic cavity sizes approach a cubic half-wavelength the spatial and spectral densities (both electronic and optical) increase to a point where the light-matter coupling becomes so strong that spontaneous emission is replaced by the coherent exchange of energy between the two systems.

The lithographic control over the wavelength and polarization supported within photonic crystal cavities can also be used to construct compact nanophotonic laser and detector arrays, as well as all-optical gates and routers. We have already demonstrated room temperature lasing in the smallest optical cavities [6], with mode volumes down to $2.5\left(\lambda / 2 \mathrm{n}_{\text {slab }}\right)^{3}$, or $0.03 \mu \mathrm{m}^{3}$ in InGaAsP emitting at $1.55 \mu \mathrm{m}$. We have also been able to tune the emission wavelength of these lasers from $1450 \mathrm{~nm}$ to $1620 \mathrm{~nm}$ within a $10 \times 10$ laser array in an area of $100 \mu \mathrm{m} \times 100 \mu \mathrm{m}$ by local lithographic modification of the cavity lengths. As the mode volumes of nano-cavities are decreased, the coupling efficiency between the spontaneous emission within the cavity and the lasing mode can be significantly improved [7]. We have calculated spontaneous emission coupling factors $(\beta)$ above $85 \%$ for optimized photonic crystal lasers constructed in active quantum well material [8]. This spontaneous emission coupling efficiency can be even higher if the linewidth of the semiconductor emission is narrowed, as will be the case when using quantum dot active material. Therefore, single

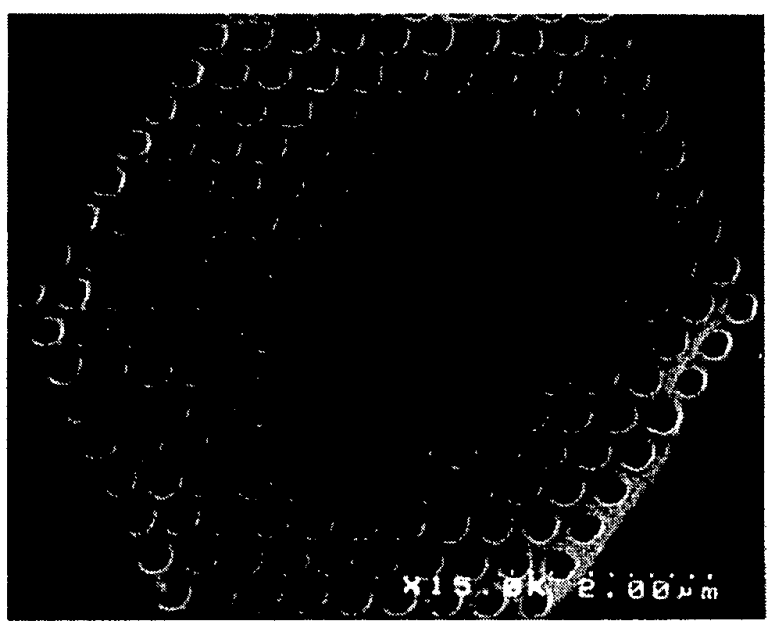

Figure 4. Multiple cavities fabricated on a Si wafer. defect photonic crystal lasers represent in many ways the ultimate evolution of VCSELs, since control over both vertical and lateral spontaneous emission is possible. With most of the spontaneous emission funneled into a single optical mode, the photonic crystal laser can be modulated at much higher frequencies even close to threshold. The photonic crystal provides us with the unique opportunity of coupling light emitted by one cavity, and using it to optically pump another with negligible diffraction losses. Photonic crystals are also the perfect medium for constructing what have been termed "photonic molecules", or interconnected cavities that 
can share and exchange photons. Finally, we have shown that the emission wavelength of light from these photonic crystal lasers can be varied by simple adjustments of the lithographic pattern during their fabrication. Thus, single mode lasers emitting at $1450 \mathrm{~nm}$ can be fabricated only microns apart from lasers emitting at $1600 \mathrm{~nm}$, and could share the same waveguide slab. Photonic crystals provide us with the opportunity of constructing very compact laser sources with designed frequencies (Figure 3) and polarization as well as wavelength and polarization sensitive detector arrays. Moreover, they can form very flexible platforms for connecting optical sources, detectors, routers, modulators, polarizers and filters in very compact microfabricated systems.

At $\sim 1.5 \mu \mathrm{m}$ wavelengths, it is possible to use silicon as a low-absorption waveguide material, and to leverage from the extensive fabrication and wafer preparation experience of the microelectronics industry. In particular, semiconductor on insulator (SOI), such as Smartcut and SIMOX structures lend themselves well to fabrication of single mode waveguides from high index silicon and the fabrication of passive 2D photonic crystal structures. In one commercially available SOI process, called Smartcut, it is possible to form a well-defined silicon dioxide layer underneath a thin slab of single crystal silicon layer at the surface (Figure 6). When designed properly, this slab can serve as a high index optical waveguide, and can easily be patterned to define 2D PBG material.
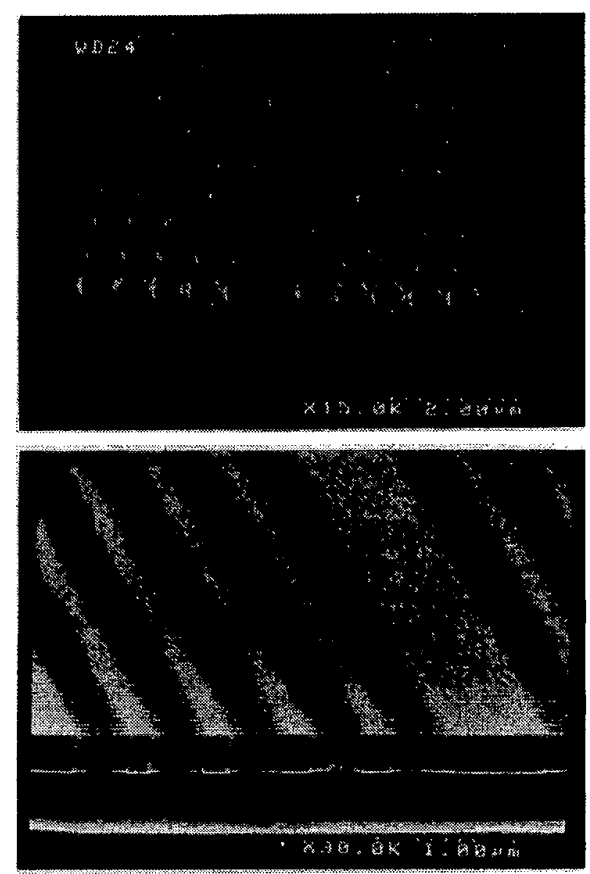

Photonic crystal waveguides play a crucial role in photonic crystal integrated circuits. These waveguides are responsible for transferring light throughout the integrated circuit as well as for the coupling of light into and out of the integrated circuit. Therefore, a careful

Figure 5. Angle view and crossection through a suspended photonic bandgap waveguide fabricated from SOI silicon. investigation of the properties of these waveguides is an important of our research. Our goal is to combine theoretical analysis and optimization with experimental techniques to develop a systematic method for design of the waveguides and control of the properties of the waveguide modes to obtain the optimum performance for the desired application.

[1] J.L. Jewell, J.P. Harbison, A. Scherer, Y.H. Lee, L.T. Florez, "Vertical cavity surface emitting lasers: Growth, Design, Fabrication, Measurements", IEEE J. Quantum Electronics, vol. 27, p1332 (1991), A. Scherer, J.L. Jewell, J.P. Harbison, "Lasing in sub-micron wide vertical cavity microlasers", Opt. Phot. News, 2, p9, (1991).

[2] E. Yablonovitch, "Inhibited spontaneous emission in solid state physics and 
electronics?, Phys. Rev. Lett., vol. 58, p2059 (1987).

[3] S. John, "Strong localization of photons in certain disordered dielectric superlattices", Phys. Rev. Lett., vol. 58, p2486, (1997).

[4] P. St. J. Russell, D.M. Atkin, T.A. Birks, Bound Modes of Two-Dimensional photonic crystal waveguides, p 203, Kluver academic publishers, The Netherlands, (1996).

[5] P.R. Villeneuve, S. Fan, S.G. Johnson, J.D. Joannopoulos, "Three-dimensional photon confinement in photonic crystals of low-dimensional preiodicity", IEEE Proc. Optoelectronics., vol. 145, p384, (1997).

[6] O. Painter, A. Husain, A. Scherer, J.D. O’Brien, I. Kim, P.D. Dapkus, "Room Temperature Photonic Crystal Defect Lasers at Near-infrared wavelengths in InGaAsP, J. Lightwave Technology, vol.17, p 2082, (1999).

[7] E.M. Purcell, " Spontaneous emission probablies at radio frequencies", Phys Rev., vol. 69, p681, (1946).

[8] J. Vuckovich, O. Painter, Y. Xu, A. Yariv, A. Scherer, "Finite Difference time domain calculations of the spontaneous emission coupling factor in optical microcavities", IEEE J. of Quantum Electronics, vol. 35, p 1168, (1999). 